\title{
PPARGC1A rs3736265 G>A polymorphism is associated with decreased risk of type 2 diabetes mellitus and fasting plasma glucose level
}

\author{
Li Zhu', ${ }^{1,}$, Qiuyu Huang ${ }^{2, *}$, Zhiqiang Xie ${ }^{3, *}$, Mingqiang Kang ${ }^{4}$, Hao Ding ${ }^{5}$, Boyang \\ Chen $^{4}$, Yu Chen ${ }^{6}$, Chao Liu', Yafeng Wang ${ }^{8}$ and Weifeng Tang ${ }^{4}$ \\ ${ }^{1}$ Department of Nephrology, Affiliated People's Hospital of Jiangsu University, Zhenjiang, Jiangsu Province, China \\ ${ }^{2}$ Department of Cardiac Surgery, Fujian Medical University Union Hospital, Fuzhou, Fujian Province, China \\ ${ }^{3}$ Department of Clinical Laboratory, Fujian Medical University Union Hospital, Fuzhou, Fujian Province, China \\ ${ }^{4}$ Department of Thoracic Surgery, Fujian Medical University Union Hospital, Fuzhou, Fujian Province, China \\ ${ }^{5}$ Department of Respiratory Disease, Affiliated People's Hospital of Jiangsu University, Zhenjiang, Jiangsu Province, China \\ ${ }^{6}$ Department of Medical Oncology, Fujian Provincial Cancer Hospital, Fujian Medical University Cancer Hospital, Fuzhou, \\ Fujian Province, China \\ ${ }^{7}$ Department of Cardiothoracic Surgery, Affiliated People's Hospital of Jiangsu University, Zhenjiang, Jiangsu Province, China \\ ${ }^{8}$ Department of Cardiology, The People's Hospital of Xishuangbanna Dai Autonomous Prefecture, Jinghong, Yunnan Province, \\ China \\ "These authors have contributed equally to this work \\ Correspondence to: Weifeng Tang, email: twf001001@126.com \\ Yafeng Wang, email: wangyafeng0910@126.com \\ Keywords: PPARG, PPARGCIA, PPARGC1B, polymorphism, type 2 diabetes mellitus \\ Received: January 05, $2017 \quad$ Accepted: February 13, $2017 \quad$ Published: March 17, 2017 \\ Copyright: Zhu et al. This is an open-access article distributed under the terms of the Creative Commons Attribution License \\ (CC-BY), which permits unrestricted use, distribution, and reproduction in any medium, provided the original author and source \\ are credited.
}

\section{ABSTRACT}

It has been reported that peroxisome proliferator-activated receptor gamma (PPARG) and peroxisome proliferator-activated receptor gamma co-activator 1 (PPARGC1) family (e.g. PPARGC1A and PPARGC1B) are key agents in the development and pathophysiology of type 2 diabetes mellitus (T2DM). In this study, we designed a case-control study and selected PPARG rs1801282 C>G, PPARG rs3856806 C>T, PPARGC1A rs8192678 C>T, PPARGC1A rs2970847 C>T, PPARGC1A rs3736265 G>A, PPARGC1B rs7732671 G>C and PPARGC1B rs17572019 G $>$ A polymorphisms to assess the relationship between these polymorphisms and T2DM using the SNPscan method. $A$ total of 502 T2DM patients and 784 non-diabetic controls were enrolled. We found that PPARGC1A rs3736265 G >A polymorphism was correlated with a borderline decreased susceptibility of T2DM. In a subgroup analysis by age, sex, alcohol use, smoking status and body mass index, a significantly decreased risk of T2DM in <65 years and female groups was found. Haplotype comparison analysis indicated that CTTCGGG and CTCTGGG haplotypes with the order of PPARG rs1801282 C>G, PPARG rs3856806 C>T, PPARGC1A rs8192678 C>T, PPARGC1A rs2970847 C>T, PPARGC1A rs3736265 G>A, PPARGC1B rs7732671 G>C and PPARGC1B rs17572019 G>A polymorphisms in gene position significantly increased the risk of T2DM. However, CCCCACA haplotype conferred a decreased risk to T2DM. We also found that PPARGC1A rs3736265 A allele decreased the level of fasting plasma glucose (FPG), while increased the level of Triglyceride. In conclusion, Our findings suggest that variants of PPARGC1A rs3736265 G>A polymorphism decrease the level of FPG, improving the expectation of study in individual's prevention strategies to T2DM. 


\section{INTRODUCTION}

Type 2 diabetes mellitus (T2DM) is a most common form of diabetes and is a major public health threat. It is estimated that the prevalence of T2DM in Chinese adult is about $11.6 \%$ [1]. T2DM appears to be increasing dramatically worldwide and the vital susceptibility factors contributing to this phenomenon are poor diet, obesity, and sedentary lifestyle $[2,3]$. It is reported that both environmental risk factors and genetic components play important roles in the etiology and pathogenesis of T2DM.

T2DM, a complex metabolic disorder, is characterized by hyperglycemia with varying degrees of impaired insulin secretion and insulin resistance (IR) as a result of pancreatic $\beta$-cell dysfunction. Imbalance of energy metabolism is considered to be one of the important pathophysiological changes in T2DM, a disease which is also characterised by IR and hyperglycaemia. Accumulating evidence suggests that dysfunction of adipose tissue is contributing to the development of IR and T2DM. Obesity represents a situation of increased fat accumulation, whereas lipodystrophy indicates a situation in which the capacity of retaining lipid in adipocytes is impaired, and then prevents the accumulation of fat. In these situations, the ability of retaining lipid in adipose tissue is impaired, leading to lipotoxicity and consequently developing peripheral IR [4]. There is also evidence that saturated fatty acids are stored in non-adipocyte, decrease glucose conversion into glycogen, and result in cellular damage as a sequence of their lipotoxicity [5]. The lipotoxicity, in the $\beta$-cell, has also been considered to contribute to the etiology and pathology of T2DM [6].

In view of that, a number of studies highlighted the vital roles of energy metabolism relative genetics in determining T2DM risk; understanding single nucleotide polymorphisms (SNPs) correlated with T2DM susceptibility may be helpful for providing personalized diagnosis and prevention. The peroxisome proliferator-activated receptor gamma (PPARG), an important transcription factor, keep the balance of energy metabolism by promoting either energy dissipation or energy deposition [7]. Recent studies reported that PPARG gene was correlated with higher risk to diabetes [8] and the $\mathrm{G}$ allele of the rs $1801282 \mathrm{C}>\mathrm{G}$ polymorphism in PPARG gene was associated with T2DM rsk in a genome-wide association study (GWAS) [9] and has been replicated in some case-control studies; however, other studes found no association between this polymorphism and T2DM [10-12]. The peroxisome proliferator-activated receptor gamma co-activator 1 (PPARGC1) family (e.g. PPARGC1A, PPARGC1B) has been considered as a vital regulator of fatty acid oxidation, gluconeogenesis and adaptive thermogenesis [13]. Of late, several studies explored the association between PPARGC1A polymorphisms and risk of T2DM. Results of a pooledanalysis suggested that PPARGC1A rs8192678G $>A$ and rs $2970847 \mathrm{C}>\mathrm{T}$ polymorphisms were associated with the increased risk of T2DM in the Indian population [14, 15]. However, in these studies, the number of eligible publications and included subjects was limited and the power might be insufficient. Previous study reported that PPARGC1B rs $7732671 \mathrm{G}>\mathrm{C}$ and $\mathrm{rs} 17572019 \mathrm{G}>\mathrm{A}$ variants were associated with the decreased risk of obesity [16]. Thus, they may alter the risk of T2DM. Therefore, in this study, we designed a hospital-based case-control study and selected $P P A R G$ rs $1801282 \mathrm{C}>\mathrm{G}, P P A R G$ rs $3856806 \mathrm{C}>\mathrm{T}$, PPARGC1A rs $8192678 \mathrm{C}>\mathrm{T}, P P A R G C 1 A$ rs $2970847 \mathrm{C}>\mathrm{T}$, PPARGC1A rs3736265 G>A, PPARGC1B rs7732671 $\mathrm{G}>\mathrm{C}$ and $P P A R G C 1 B$ rs $17572019 \mathrm{G}>\mathrm{A}$ polymorphisms to assess the relationship between these SNPs and T2DM in an Eastern Chinese Han population using the SNPscan method.

\section{RESULTS}

\section{Baseline characteristics}

The anthropometric data, biochemistry characteristics, demographics and risk factors of all participants are listed in Table 1. As shown in Table 1, the mean \pm SD of height, weight, BMI, FPG, total cholesterol, triglyceride, HDL-C and LDL-C levels was significantly higher in the T2DM group compared with non-diabetic normal controls $(P<0.05)$. However, the mean $\pm \mathrm{SD}$ of systolic pressure and diastolic pressure was not significant. Additionally, Table 1 showed that the present study was fully matched by age, gender, alcohol use and smoking status. The primary information of PPARG rs 1801282 $\mathrm{C}>\mathrm{G}, P P A R G$ rs3856806 $\mathrm{C}>\mathrm{T}, P P A R G C 1 A$ rs 8192678 $\mathrm{C}>\mathrm{T}$, PPARGC1A rs $2970847 \mathrm{C}>\mathrm{T}$, PPARGC1A $\mathrm{rs} 3736265$ $\mathrm{G}>\mathrm{A}$, PPARGC1B rs $7732671 \mathrm{G}>\mathrm{C}$ and PPARGC1B rs17572019 G>A polymorphisms is showed in Table 2. For these SNPs, the genotyping success rate was more than $99 \%$ in all samples. Minor allele frequency (MAF) and HWE in controls are summarized in Table 2.

\section{Association of PPARG rs1801282 C $>$ G, PPARG rs3856806 C $>$ T, PPARGC1A rs8192678 C $>$ T, PPARGC1A rs2970847 C $>$ T, PPARGC1A rs3736265 G>A, PPARGC1B rs7732671 G $>C$ and PPARGC1B rs17572019 G $>A$ polymorphisms with T2DM}

The genotype distributions of PPARG rs 1801282 $\mathrm{C}>\mathrm{G}, P P A R G$ rs3856806 $\mathrm{C}>\mathrm{T}$, PPARGC1A rs8192678 $\mathrm{C}>\mathrm{T}$, PPARGC1A rs $2970847 \mathrm{C}>\mathrm{T}$, PPARGC1A rs 3736265 $\mathrm{G}>\mathrm{A}$, PPARGC1B rs7732671 $\mathrm{G}>\mathrm{C}$ and PPARGC1B rs17572019 G>A polymorphisms are listed in Table 3. The genotype distributions of these polymorphisms in controls were suggested to be in HWE. In the analysis of PPARGC1A rs $3736265 \mathrm{G}>\mathrm{A}$ polymorphism, differences in the frequency distribution of the GA/ 
Table 1: Distribution of selected demographic variables and risk factors in type 2 diabetes cases and controls

\begin{tabular}{|c|c|c|c|c|c|}
\hline \multirow{2}{*}{ Variable } & \multicolumn{2}{|c|}{ Cases $(n=502)$} & \multicolumn{2}{|c|}{ Controls $(n=782)$} & \multirow{2}{*}{$P^{a}$} \\
\hline & $\mathbf{n}$ & $\%$ & $\mathbf{n}$ & $\%$ & \\
\hline Age (years) & $65.20( \pm 9.51)$ & & $64.67( \pm 9.80)$ & & 0.347 \\
\hline Age (years) & & & & & 0.113 \\
\hline$<65$ & 227 & 45.22 & 389 & 49.74 & \\
\hline$\geq 65$ & 275 & 54.78 & 393 & 50.26 & \\
\hline Sex & & & & & 0.819 \\
\hline Male & 332 & 66.14 & 522 & 66.75 & \\
\hline Female & 170 & 33.86 & 260 & 33.25 & \\
\hline Smoking status & & & & & 0.264 \\
\hline Never & 333 & 66.33 & 542 & 69.31 & \\
\hline Ever & 169 & 33.67 & 240 & 30.69 & \\
\hline Alcohol use & & & & & 0.263 \\
\hline Never & 453 & 90.24 & 690 & 88.24 & \\
\hline Ever & 49 & 9.76 & 92 & 11.76 & \\
\hline Height (m) & $1.68( \pm 0.08)$ & & $1.66( \pm 0.07)$ & & 0.015 \\
\hline Weight (kg) & $67.63( \pm 11.42)$ & & $64.62( \pm 9.96)$ & & $<0.001$ \\
\hline BMI $\left(\mathrm{kg} / \mathrm{m}^{2}\right)$ & $24.95( \pm 3.64)$ & & $23.51( \pm 2.94)$ & & $<0.001$ \\
\hline BMI $\left(\mathrm{kg} / \mathrm{m}^{2}\right)$ & & & & & $<0.001$ \\
\hline$<24$ & 210 & & 436 & & \\
\hline$\geq 24$ & 292 & & 346 & & \\
\hline $\begin{array}{l}\text { Systolic pressure } \\
(\mathrm{mmHg})\end{array}$ & $135.08( \pm 17.83)$ & & $134.02( \pm 17.71)$ & & 0.297 \\
\hline $\begin{array}{l}\text { Diastolic pressure } \\
(\mathrm{mmHg})\end{array}$ & $79.79( \pm 10.35)$ & & $80.06( \pm 10.02)$ & & 0.649 \\
\hline $\mathrm{FPG}(\mathrm{mmol} / \mathrm{L})$ & $8.08( \pm 2.76)$ & & $5.13( \pm 0.49)$ & & $<0.001$ \\
\hline $\begin{array}{l}\text { Total cholesterol } \\
(\mathrm{mmol} / \mathrm{L})\end{array}$ & $4.61( \pm 1.24)$ & & $4.88( \pm 1.02)$ & & $<0.001$ \\
\hline Triglyceride (mmol/L) & $1.74( \pm 1.14)$ & & $1.55( \pm 0.96)$ & & 0.001 \\
\hline HDL-C (mmol/L) & $1.13( \pm 0.37)$ & & $1.30( \pm 0.37)$ & & $<0.001$ \\
\hline LDL-C (mmol/L) & $3.00( \pm 1.07)$ & & $3.14( \pm 0.82)$ & & 0.010 \\
\hline
\end{tabular}

a Two-sided $x^{2}$ test and student $\mathrm{t}$ test; Bold values are statistically significant $(P<0.05)$; BMI, body mass index; FPG, fasting plasma glucose; HDL-C, high-density lipoprotein cholesterol; LDL-C, low-density lipoprotein cholesterol.

AA genotypes compared with the GG genotype and GA genotype compared with the GG genotype between T2DM patients and non-diabetic controls were found [GA+AA vs. GG: crude odds ratio $(\mathrm{OR})=0.76,95 \%$ confidence interval $(\mathrm{CI})=0.59-0.99, P=0.041$ and GA vs. GG: crude $\mathrm{OR}=0.76,95 \% \mathrm{CI}=0.58-1.00, P$ $=0.049$ (Table 3)]. However, PPARG rs1801282 C $>\mathrm{G}$,
$P P A R G$ rs3856806 $\mathrm{C}>\mathrm{T}, P P A R G C 1 A$ rs8192678 $\mathrm{C}>\mathrm{T}$, $P P A R G C 1 A$ rs $2970847 \mathrm{C}>\mathrm{T}, P P A R G C 1 B$ rs $7732671 \mathrm{G}>\mathrm{C}$ and $P P A R G C 1 B$ rs $17572019 \mathrm{G}>\mathrm{A}$ polymorphisms was not associated with T2DM susceptibility (Table 3 ). In two genetic models, logistic regression analysis demonstrated that PPARGC1A rs3736265 $\mathrm{G}>\mathrm{A}$ polymorphism was associated with a borderline statistically risk of T2DM. 
Table 2: Primary information for $P P A R G$ rs1801282 C $>\mathrm{G}, P P A R G$ rs3856806 C $>\mathrm{T}, P P A R G C 1 A$ rs8192678 C $>\mathrm{T}$, PPARGC1A rs2970847 C>T, PPARGC1A rs3736265 G>A, PPARGC1B rs7732671 G>C and PPARGC1B rs17572019 G>A polymorphisms

\begin{tabular}{|c|c|c|c|c|c|c|c|}
\hline $\begin{array}{l}\text { Genotyped } \\
\text { SNPs }\end{array}$ & $\begin{array}{c}P P A R G \\
\text { rs1801282 } \\
\text { C }>G\end{array}$ & $\begin{array}{c}P P A R G \\
\text { rs3856806 } \\
\text { C }>T\end{array}$ & $\begin{array}{c}P P A R G C 1 A \\
\text { rs8192678 } \\
\text { C }>\text { T }\end{array}$ & $\begin{array}{c}P P A R G C 1 A \\
\text { rs2970847 } \\
C>T \\
\end{array}$ & $\begin{array}{c}P P A R G C 1 A \\
\text { rs3736265 } \\
\mathrm{G}>\mathrm{A}\end{array}$ & $\begin{array}{c}P P A R G C 1 B \\
\text { rs } 7732671 \\
G>C\end{array}$ & $\begin{array}{c}P P A R G C 1 B \\
\text { rs17572019 } \\
\text { G }>A\end{array}$ \\
\hline Chromosome & 3 & 3 & 4 & 4 & 4 & 5 & 5 \\
\hline Function & missense & $\begin{array}{c}\text { coding- } \\
\text { synonymous }\end{array}$ & missense & $\begin{array}{c}\text { coding- } \\
\text { synonymous }\end{array}$ & missense & missense & missense \\
\hline $\begin{array}{l}\text { Chr Pos (NCBI } \\
\text { Build 37) }\end{array}$ & 12393125 & 12475557 & 23815662 & 23815924 & 23814707 & 149212243 & 149212471 \\
\hline $\begin{array}{l}\text { Regulome DB } \\
\text { Score }^{\mathrm{a}}\end{array}$ & - & $2 b$ & 6 & - & 6 & 5 & 5 \\
\hline $\begin{array}{l}\mathrm{MAF}^{\mathrm{b}} \text { for } \\
\text { Chinese in } \\
\text { database }\end{array}$ & 0.07 & 0.25 & 0.35 & 0.28 & 0.23 & 0.09 & 0.07 \\
\hline $\begin{array}{l}\text { MAF in our } \\
\text { controls }(n= \\
782)\end{array}$ & 0.05 & 0.22 & 0.44 & 0.21 & 0.16 & 0.06 & 0.06 \\
\hline $\begin{array}{l}P \text { value for } \\
\mathrm{HWE}^{\mathrm{c}} \text { test in } \\
\text { our controls }\end{array}$ & 0.973 & 0.381 & 0.850 & 0.281 & 0.064 & 0.693 & 0.305 \\
\hline $\begin{array}{l}\text { Genotyping } \\
\text { method }\end{array}$ & SNPscan & SNPscan & SNPscan & SNPscan & SNPscan & SNPscan & SNPscan \\
\hline $\begin{array}{l}\% \text { Genotyping } \\
\text { value }\end{array}$ & $99.61 \%$ & $99.61 \%$ & $99.61 \%$ & $99.61 \%$ & $99.38 \%$ & $99.61 \%$ & $99.61 \%$ \\
\hline
\end{tabular}

${ }^{a}$ http://www.regulomedb.org/;

${ }^{\mathrm{b}} \mathrm{MAF}$ : minor allele frequency;

${ }^{c}$ HWE: Hardy-Weinberg equilibrium;

Table 3: Logistic regression analyses of associations between $P P A R G$ rs1801282 $\mathrm{C}>\mathrm{G}, P P A R G$ rs3856806 C $>\mathrm{T}$, PPARGC1A rs8192678 C $>\mathrm{T}$, PPARGC1A rs2970847 C $>\mathrm{T}$, PPARGC1A rs3736265 G>A, PPARGC1B rs $7732671 \mathrm{G}>\mathrm{C}$ and $P P A R G C 1 B$ rs17572019 $\mathrm{G}>\mathrm{A}$ polymorphisms and risk of type 2 diabetes

\begin{tabular}{|c|c|c|c|c|c|c|c|c|}
\hline \multirow[t]{2}{*}{ Genotype } & \multicolumn{2}{|c|}{$\begin{array}{c}\text { Cases } \\
(n=502)\end{array}$} & \multicolumn{2}{|c|}{$\begin{array}{c}\text { Controls } \\
(n=782)\end{array}$} & \multirow{2}{*}{$\begin{array}{c}\text { Crude OR } \\
(95 \% \mathrm{CI})\end{array}$} & \multirow[t]{2}{*}{$P$} & \multirow{2}{*}{$\begin{array}{l}\text { Adjusted OR }{ }^{\mathrm{a}} \\
(95 \% \mathrm{CI})\end{array}$} & \multirow[t]{2}{*}{$P$} \\
\hline & $\mathbf{n}$ & $\%$ & n & $\%$ & & & & \\
\hline \multicolumn{9}{|c|}{$\begin{array}{l}P P A R G \\
\text { rs } 1801282 \mathrm{C}>\mathrm{G}\end{array}$} \\
\hline $\mathrm{CC}$ & 457 & 91.95 & 704 & 90.03 & 1.00 & & 1.00 & \\
\hline $\mathrm{CG}$ & 40 & 8.05 & 76 & 9.72 & $0.80(0.54-1.20)$ & 0.280 & $0.76(0.50-1.14)$ & 0.179 \\
\hline GG & 0 & 0 & 2 & 0.26 & - & - & - & - \\
\hline $\mathrm{GC}+\mathrm{GG}$ & 40 & 8.05 & 78 & 9.98 & $0.79(0.53-1.18)$ & 0.247 & $0.75(0.50-1.12)$ & 0.164 \\
\hline $\mathrm{CC}+\mathrm{GC}$ & 497 & 100 & 780 & 99.75 & 1.00 & & 1.00 & \\
\hline GG & 0 & 0 & 2 & 0.26 & - & - & - & - \\
\hline $\mathrm{G}$ allele & 40 & 4.02 & 80 & 5.12 & & & & \\
\hline
\end{tabular}

(Continued) 


\begin{tabular}{|c|c|c|c|c|c|c|c|c|}
\hline \multirow[t]{2}{*}{ Genotype } & \multicolumn{2}{|c|}{$\begin{array}{c}\begin{array}{c}\text { Cases } \\
(\mathrm{n}=502)\end{array} \\
\end{array}$} & \multicolumn{2}{|c|}{$\begin{array}{c}\text { Controls } \\
(\mathrm{n}=782)\end{array}$} & \multirow{2}{*}{$\begin{array}{c}\text { Crude OR } \\
(95 \% \mathrm{CI})\end{array}$} & \multirow[t]{2}{*}{$P$} & \multirow{2}{*}{$\begin{array}{l}\text { Adjusted OR }{ }^{\mathrm{a}} \\
\quad(95 \% \mathrm{CI})\end{array}$} & \multirow[t]{2}{*}{$P$} \\
\hline & $\mathbf{n}$ & $\%$ & $\mathbf{n}$ & $\%$ & & & & \\
\hline \multicolumn{9}{|c|}{$\begin{array}{l}\text { PPARG } \\
\text { rs3856806 C>T }\end{array}$} \\
\hline $\mathrm{CC}$ & 278 & 55.94 & 474 & 60.61 & 1.00 & & 1.00 & \\
\hline CT & 196 & 39.44 & 275 & 35.17 & $1.19(0.94-1.51)$ & 0.140 & $1.17(0.92-1.48)$ & 0.204 \\
\hline TT & 23 & 4.63 & 33 & 4.22 & $1.17(0.67-2.03)$ & 0.583 & $1.19(0.68-2.08)$ & 0.541 \\
\hline $\mathrm{CT}+\mathrm{TT}$ & 219 & 44.06 & 308 & 39.39 & $1.21(0.97-1.52)$ & 0.098 & $1.19(0.94-1.50)$ & 0.140 \\
\hline $\mathrm{CC}+\mathrm{CT}$ & 474 & 95.37 & 749 & 95.78 & 1.00 & & 1.00 & \\
\hline $\mathrm{TT}$ & 23 & 4.63 & 33 & 4.22 & $1.10(064-1.90)$ & 0.728 & $1.14(0.65-1.97)$ & 0.653 \\
\hline $\mathrm{T}$ allele & 242 & 24.35 & 341 & 21.80 & & & & \\
\hline \multicolumn{9}{|c|}{$\begin{array}{l}\text { PPARGC1A } \\
\text { rs8192678 C }>\mathrm{T}\end{array}$} \\
\hline $\mathrm{CC}$ & 138 & 27.77 & 250 & 31.97 & 1.00 & & 1.00 & \\
\hline CT & 251 & 50.50 & 382 & 48.85 & $1.15(0.89-1.49)$ & 0.296 & $1.12(0.86-1.46)$ & 0.402 \\
\hline TT & 108 & 21.73 & 150 & 19.18 & $1.26(0.91-1.74)$ & 0.161 & $1.22(0.88-1.69)$ & 0.240 \\
\hline $\mathrm{CT}+\mathrm{TT}$ & 269 & 54.12 & 532 & 68.03 & $1.22(0.96-1.57)$ & 0.111 & $\begin{array}{c}1.19(0.93- \\
1.1 .53)\end{array}$ & 0.178 \\
\hline $\mathrm{CC}+\mathrm{CT}$ & 389 & 78.27 & 632 & 80.82 & 1.00 & & 1.00 & \\
\hline TT & 108 & 21.73 & 150 & 19.18 & $1.17(0.89-1.54)$ & 0.269 & $1.15(0.87-1.52)$ & 0.334 \\
\hline $\mathrm{T}$ allele & 467 & 46.98 & 682 & 43.61 & & & & \\
\hline \multicolumn{9}{|c|}{$\begin{array}{l}P P A R G C 1 A \\
\text { rs } 2970847 \mathrm{C}>\mathrm{T}\end{array}$} \\
\hline $\mathrm{CC}$ & 310 & 62.37 & 485 & 62.02 & 1.00 & & 1.00 & \\
\hline CT & 160 & 32.19 & 268 & 34.27 & $0.92(0.72-1.17)$ & 0.495 & $0.93(0.73-1.19)$ & 0.582 \\
\hline TT & 27 & 5.43 & 29 & 3.71 & $1.43(0.83-2.47)$ & 0.194 & $1.50(0.87-2.60)$ & 0.148 \\
\hline $\mathrm{CT}+\mathrm{TT}$ & 187 & 37.63 & 297 & 37.98 & $0.99(0.78-1.24)$ & 0.899 & $1.00(0.79-1.27)$ & 0.979 \\
\hline $\mathrm{CC}+\mathrm{CT}$ & 470 & 94.57 & 753 & 96.29 & 1.00 & & 1.00 & \\
\hline TT & 27 & 5.43 & 29 & 3.71 & $1.49(0.87-2.55)$ & 0.144 & $1.55(0.90-2.68)$ & 0.112 \\
\hline $\mathrm{T}$ allele & 214 & 21.53 & 326 & 20.84 & & & & \\
\hline \multicolumn{9}{|c|}{$\begin{array}{l}\text { PPARGC1A } \\
\text { rs3736265 G>A }\end{array}$} \\
\hline GG & 380 & 76.61 & 557 & 71.41 & 1.00 & & & \\
\hline GA & 103 & 20.77 & 196 & 25.13 & $0.76(0.58-1.00)$ & 0.049 & $0.76(0.58-1.00)$ & 0.053 \\
\hline AA & 13 & 2.62 & 27 & 3.46 & $0.70(0.36-1.37)$ & 0.295 & $0.74(0.37-1.46)$ & 0.378 \\
\hline $\mathrm{GA}+\mathrm{AA}$ & 116 & 23.39 & 223 & 28.59 & $0.76(0.59-0.99)$ & 0.041 & $0.77(0.59-1.00)$ & 0.050 \\
\hline $\mathrm{GG}+\mathrm{GA}$ & 483 & 97.38 & 753 & 96.54 & 1.00 & & 1.00 & \\
\hline AA & 13 & 2.62 & 27 & 3.46 & $0.75(0.38-1.47)$ & 0.403 & $0.79(0.40-1.56)$ & 0.494 \\
\hline A allele & 129 & 13.00 & 250 & 15.98 & & & & \\
\hline
\end{tabular}

(Continued) 


\begin{tabular}{|c|c|c|c|c|c|c|c|c|}
\hline \multirow{2}{*}{ Genotype } & \multicolumn{2}{|c|}{$\begin{array}{c}\text { Cases } \\
(\mathrm{n}=502)\end{array}$} & \multicolumn{2}{|c|}{$\begin{array}{l}\text { Controls } \\
(n=782)\end{array}$} & \multirow{2}{*}{$\begin{array}{c}\text { Crude OR } \\
(95 \% \mathrm{CI})\end{array}$} & \multirow[t]{2}{*}{$P$} & \multirow{2}{*}{$\begin{array}{l}\text { Adjusted OR }{ }^{\mathrm{a}} \\
\quad(95 \% \mathrm{CI})\end{array}$} & \multirow[t]{2}{*}{$P$} \\
\hline & $\mathbf{n}$ & $\%$ & $\mathbf{n}$ & $\%$ & & & & \\
\hline \multicolumn{9}{|c|}{$\begin{array}{l}\text { PPARGC1B } \\
\text { rs7732671 G>C }\end{array}$} \\
\hline GG & 435 & 87.53 & 698 & 89.26 & 1.00 & & 1.00 & \\
\hline $\mathrm{GC}$ & 61 & 12.27 & 81 & 10.36 & $1.20(0.84-1.70)$ & 0.323 & $1.20(0.84-1.72)$ & 0.314 \\
\hline $\mathrm{CC}$ & 1 & 0.20 & 3 & 0.38 & $0.53(0.06-5.10)$ & 0.582 & $0.48(0.05-4.71)$ & 0.527 \\
\hline $\mathrm{GC}+\mathrm{CC}$ & 62 & 12.47 & 84 & 10.74 & $1.18(0.84-1.68)$ & 0.342 & $1.19(0.83-1.69)$ & 0.341 \\
\hline $\mathrm{GG}+\mathrm{GC}$ & 496 & 99.80 & 779 & 99.62 & 1.00 & & 1.00 & \\
\hline $\mathrm{CC}$ & 1 & 0.20 & 3 & 0.38 & $0.52(0.05-5.05)$ & 0.576 & $0.47(0.05-4.66)$ & 0.520 \\
\hline $\mathrm{C}$ allele & 63 & 6.34 & 87 & 5.56 & & & & \\
\hline \multicolumn{9}{|c|}{$\begin{array}{l}P P A R G C 1 B \\
\text { rs17572019 } \\
\mathrm{G}>\mathrm{A}\end{array}$} \\
\hline GG & 435 & 87.53 & 698 & 89.26 & 1.00 & & & \\
\hline GA & 60 & 12.07 & 80 & 10.23 & $1.19(0.83-1.70)$ & 0.338 & $1.19(0.83-1.71)$ & 0.338 \\
\hline AA & 2 & 0.40 & 4 & 0.51 & $0.79(0.15-4.35)$ & 0.790 & $0.81(0.14-4.52)$ & 0.808 \\
\hline $\mathrm{GA}+\mathrm{AA}$ & 62 & 12.47 & 84 & 10.74 & $1.18(0.84-1.68)$ & 0.343 & $1.19(0.83-1.69)$ & 0.341 \\
\hline $\mathrm{GG}+\mathrm{GA}$ & 495 & 99.60 & 778 & 99.49 & 1.00 & & & \\
\hline AA & 2 & 0.40 & 4 & 0.51 & $0.79(0.14-4.31)$ & 0.781 & $0.80(0.14-4.49)$ & 0.80 \\
\hline A allele & 64 & 6.44 & 88 & 5.63 & & & & \\
\hline
\end{tabular}

a Adjusted for age, sex, smoking status, alcohol use and BMI status.

Bold values are statistically significant $(P<0.05)$.

When the PPARGC1A rs3736265 GG genotypes were used as the reference group, the GA/AA and GA genotype was correlated with a borderline statistically decreased susceptibility of T2DM [GA+AA vs. GG: adjusted OR = $0.77,95 \% \mathrm{CI}=0.59-1.00, P=0.050$ and $\mathrm{GA}$ vs. GG: adjusted $\mathrm{OR}=0.76,95 \% \mathrm{CI}=0.58-1.00, P=0.053$ (Table 3)].

\section{Association of PPARGC1A rs3736265 G>A polymorphism with T2DM in Different Stratification Groups}

Table 4 showed the genotype frequencies of PPARGC1A rs3736265 $\mathrm{G}>\mathrm{A}$ polymorphism in the stratified analysis based on age, gender, alcohol use, smoking status and BMI. In female group, after adjustment for age, alcohol use, smoking status and BMI by logistic regression analysis, the GA/AA and GA genotypes of PPARGC1A rs3736265 G>A polymorphism were associated with a significantly decreased risk of T2DM compared with the GG genotype [GA+AA vs.
GG: adjusted OR $=0.46,95 \%$ CI $0.28-0.74, \mathrm{P}=0.001$ and GA vs. GG: adjusted $\mathrm{OR}=0.45,95 \% \mathrm{CI}=0.28$ $0.74, P=0.002$ (Table 4)]. In $<65$ years group, after adjustment for gender, alcohol use, smoking status and BMI by logistic regression analysis, the GA/AA and GA genotypes of PPARGC1A rs3736265 G>A polymorphism were also associated with a significantly decreased risk of T2DM compared with the GG genotype [GA+AA vs. GG: adjusted $\mathrm{OR}=0.67,95 \% \mathrm{CI} 0.46-0.98, \mathrm{P}=0.038$ and GA vs. GG: adjusted $\mathrm{OR}=0.60,95 \% \mathrm{CI}=0.40-0.90$, $P=0.013$ (Table 4)]. However, in other groups, there was no correlation between PPARGC1A rs $3736265 \mathrm{G}>\mathrm{A}$ polymorphism and the risk of T2DM $(P>0.05$; Table 4$)$.

\section{SNP haplotypes}

Using an expectation-maximization algorithm software [SHESIS program (Bio-X Inc., Shanghai, China, http://analysis.bio-x.cn/myAnalysis.php)] [17], we constructed thirteen haplotypes (Table 5). Haplotype comparison analysis indicated that CTTCGGG and 
Table 4: Stratified analyses between $P P A R G C 1 A$ rs3736265 G>A polymorphism and type 2 diabetes risk by sex, age, smoking status, alcohol consumption and BMI

\begin{tabular}{|c|c|c|c|c|c|c|c|c|c|}
\hline \multirow[b]{2}{*}{ Variable } & \multicolumn{4}{|c|}{$(\text { case/control })^{a}$} & \multicolumn{5}{|c|}{ Adjusted OR ${ }^{\mathrm{b}}(95 \%$ CI $) ; P$} \\
\hline & GG & GA & $\mathbf{A A}$ & GA/AA & GG & GA & $\mathbf{A A}$ & GA/AA & $\begin{array}{c}\text { AA vs. (GA// } \\
\text { GG) }\end{array}$ \\
\hline \multicolumn{10}{|l|}{ Sex } \\
\hline Male & $244 / 383$ & $74 / 116$ & $11 / 21$ & $85 / 137$ & 1.00 & $\begin{array}{c}0.99 \\
(0.71-1.39) \\
P: 0.952\end{array}$ & $\begin{array}{c}0.86 \\
(0.40-1.84) \\
P: 0.705\end{array}$ & $\begin{array}{c}0.98 \\
(0.71-1.35) \\
P: 0.886\end{array}$ & $\begin{array}{c}0.87 \\
(0.41-1.85) \\
P: 0.713\end{array}$ \\
\hline Female & $136 / 174$ & $29 / 80$ & $2 / 6$ & $31 / 86$ & 1.00 & $\begin{array}{c}0.45 \\
(0.28-0.74) \\
P: 0.002\end{array}$ & $\begin{array}{c}0.35 \\
(0.07-1.79) \\
P: 0.206\end{array}$ & $\begin{array}{c}0.46 \\
(0.28-0.74) \\
P: 0.001\end{array}$ & $\begin{array}{c}0.42 \\
(0.08-2.15) \\
P: 0.298\end{array}$ \\
\hline \multicolumn{10}{|l|}{ Age (years) } \\
\hline$<65$ & $169 / 264$ & $46 / 112$ & $9 / 11$ & $55 / 123$ & 1.00 & $\begin{array}{c}0.60 \\
(0.40-0.90) \\
P: 0.013\end{array}$ & $\begin{array}{c}1.28 \\
(0.51-3.24) \\
P: 0.596\end{array}$ & $\begin{array}{c}0.67 \\
(0.46-0.98) \\
\text { P: } 0.038\end{array}$ & $\begin{array}{c}1.43 \\
(0.57-3.60) \\
P: 0.442\end{array}$ \\
\hline$\geq 65$ & $211 / 293$ & $57 / 84$ & $4 / 16$ & $61 / 100$ & 1.00 & $\begin{array}{c}0.96 \\
(0.65-1.41) \\
P: 0.822\end{array}$ & $\begin{array}{c}0.33 \\
(0.11-1.03) \\
P: 0.055\end{array}$ & $\begin{array}{c}0.87 \\
(0.60-1.26) \\
P: 0.450\end{array}$ & $\begin{array}{c}0.34 \\
(0.11-1.04) \\
P: 0.058\end{array}$ \\
\hline \multicolumn{10}{|l|}{$\begin{array}{l}\text { Smoking } \\
\text { status }\end{array}$} \\
\hline Never & $251 / 386$ & $69 / 137$ & $10 / 19$ & $79 / 156$ & 1.00 & $\begin{array}{c}0.77 \\
(0.55-1.07) \\
P: 0.115\end{array}$ & $\begin{array}{c}0.79 \\
(0.36-1.75) \\
P: 0.560\end{array}$ & $\begin{array}{c}0.78 \\
(0.57-1.07) \\
P: 0.121\end{array}$ & $\begin{array}{c}0.85 \\
(0.38-1.86) \\
P: 0.677\end{array}$ \\
\hline Ever & $129 / 171$ & $34 / 59$ & $3 / 8$ & $37 / 67$ & 1.00 & $\begin{array}{c}0.70 \\
(0.43-1.16) \\
P: 0.163\end{array}$ & $\begin{array}{c}0.56 \\
(0.14-2.22) \\
P: 0.409\end{array}$ & $\begin{array}{c}0.69 \\
(0.43-1.12) \\
P: 0.132\end{array}$ & $\begin{array}{c}0.61 \\
(0.16-2.42) \\
P: 0.484\end{array}$ \\
\hline \multicolumn{10}{|l|}{ Alcohol use } \\
\hline Never & $345 / 490$ & $94 / 175$ & $9 / 24$ & $103 / 199$ & 1.00 & $\begin{array}{c}0.76 \\
(0.57-1.01) \\
P: 0.061\end{array}$ & $\begin{array}{c}0.53 \\
(0.24-1.17) \\
P: 0.118\end{array}$ & $\begin{array}{c}0.74 \\
(0.56-0.98) \\
P: 0.035\end{array}$ & $\begin{array}{c}0.57 \\
(0.26-1.26) \\
P: 0.165\end{array}$ \\
\hline Ever & $35 / 67$ & $9 / 21$ & $4 / 3$ & $13 / 24$ & 1.00 & $\begin{array}{c}0.80 \\
(0.33-1.94) \\
P: 0.620\end{array}$ & $\begin{array}{c}2.28 \\
(0.46-11.31) \\
P: 0.313\end{array}$ & $\begin{array}{c}0.99 \\
(0.44-2.21) \\
P: 0.976\end{array}$ & $\begin{array}{c}2.37 \\
(0.48-11.60) \\
P: 0.288\end{array}$ \\
\hline \multicolumn{10}{|l|}{ BMI $\left(\mathrm{kg} / \mathrm{m}^{2}\right)$} \\
\hline$<24$ & $157 / 314$ & $45 / 105$ & $6 / 15$ & $51 / 120$ & 1.00 & $\begin{array}{c}0.85 \\
(0.57-1.26) \\
P: 0.418\end{array}$ & $\begin{array}{c}0.84 \\
(0.32-2.21) \\
P: 0.722\end{array}$ & $\begin{array}{c}0.86 \\
(0.59-1.26) \\
P: 0.428\end{array}$ & $\begin{array}{c}0.88 \\
(0.33-2.30) \\
P: 0.787\end{array}$ \\
\hline$\geq 24$ & $224 / 243$ & $58 / 91$ & $7 / 12$ & $65 / 103$ & 1.00 & $\begin{array}{c}0.69 \\
(0.47-1.01) \\
P: 0.054\end{array}$ & $\begin{array}{c}0.70 \\
(0.27-1.82) \\
P: 0.460\end{array}$ & $\begin{array}{c}0.70 \\
(0.49-1.00) \\
P: 0.053\end{array}$ & $\begin{array}{c}2.37 \\
(0.29-1.98) \\
P: 0.576\end{array}$ \\
\hline
\end{tabular}

a The genotyping was successful in 502 (98.80\%) type 2 diabetes cases, and $782(99.74 \%)$ controls for PPARGC1A rs3736265 G>A;

${ }^{\mathrm{b}}$ Adjusted for age, sex, smoking status, alcohol use and BMI (besides stratified factors accordingly) in a logistic regression model;

Bold values are statistically significant $(P<0.05)$ 
Table 5: PPARG-PPARGC1A-PPARGC1B haplotype frequencies (\%) in cases and controls and risk of type 2 diabetes

\begin{tabular}{|c|c|c|c|c|}
\hline \multirow{2}{*}{ Haplotypes } & Cases $(n=1004)$ & Controls $(n=1564)$ & \multirow{2}{*}{ Crude OR (95\% CI) } & \multirow{2}{*}{$\boldsymbol{P}$} \\
\hline & n (\%) & n (\%) & & \\
\hline $\mathrm{C} \mathrm{C} \mathrm{T} \mathrm{C} \mathrm{G} \mathrm{G} \mathrm{G}$ & $329(33.13)$ & $507(32.50)$ & 1.00 & \\
\hline $\mathrm{C} \mathrm{C} \mathrm{C} \mathrm{T} \mathrm{G} \mathrm{G} \mathrm{G}$ & 133(13.39) & $244(15.64)$ & $0.84(0.65-1.08)$ & 0.176 \\
\hline $\mathrm{C} \mathrm{C} \mathrm{C} \mathrm{C} \mathrm{G} \mathrm{G} \mathrm{G}$ & $129(13.00)$ & $200(12.82)$ & $0.99(0.77-1.29)$ & 0.964 \\
\hline $\mathrm{CCCCAG}$ & $98(9.82)$ & $188(12.05)$ & $0.80(0.61-1.06)$ & 0.126 \\
\hline C T T C G G G & $91(9.16)$ & $101(6.47)$ & $1.39(1.01-1.90)$ & 0.041 \\
\hline C T C T G G G & $57(5.74)$ & $48(3.08)$ & $1.83(1.22-2.75)$ & 0.003 \\
\hline $\mathrm{C}$ T C C G G & $32(3.22)$ & $72(4.62)$ & $0.68(0.44-1.06)$ & 0.090 \\
\hline $\mathrm{C} \mathrm{C} \mathrm{T} \mathrm{C} \mathrm{G} \mathrm{C} \mathrm{A}$ & $31(3.12)$ & $32(2.05)$ & $1.49(0.89-2.49)$ & 0.124 \\
\hline C T C C A G G & $24(2.42)$ & $40(2.56)$ & $0.92(0.55-1.56)$ & 0.770 \\
\hline G T T C G G G & $15(1.51)$ & $27(1.73)$ & $0.86(0.45-1.63)$ & 0.637 \\
\hline $\mathrm{C} \mathrm{C} \mathrm{C} \mathrm{C} \mathrm{G} \mathrm{C}$ & $13(1.31)$ & $16(1.03)$ & $1.25(0.59-2.64)$ & 0.553 \\
\hline G T C T G G G & 11(1.11) & $15(0.96)$ & $1.13(0.51-2.49)$ & 0.762 \\
\hline $\mathrm{C} \mathrm{C} \mathrm{C} \mathrm{C} \mathrm{A} \mathrm{C} \mathrm{A}$ & $0(0)$ & $10(0.64)$ & - & 0.008 \\
\hline Others & $30(3.02)$ & $60(3.85)$ & $0.77(.49-1.22)$ & 0.265 \\
\hline
\end{tabular}

With the order of PPARG rs $1801282 \mathrm{C}>\mathrm{G}, P P A R G \mathrm{rs} 3856806 \mathrm{C}>\mathrm{T}, P P A R G C 1 A$ rs8192678 C $>\mathrm{T}, P P A R G C 1 A$ rs 2970847 $\mathrm{C}>\mathrm{T}, P P A R G C 1 A$ rs3736265 G>A, PPARGC1B rs7732671 G>C and PPARGC1B rs17572019 G>A polymorphisms in gene position. Bold values are statistically significant $(P<0.05)$.

CTCTGGG haplotypes with the order of $P P A R G$ rs1801282 $\mathrm{C}>\mathrm{G}, P P A R G$ rs3856806 C $>\mathrm{T}, P P A R G C 1 A$ rs8192678 C $>$ T, PPARGC1A rs $2970847 \mathrm{C}>\mathrm{T}, P P A R G C 1 A$ rs3736265 $\mathrm{G}>\mathrm{A}, P P A R G C 1 B$ rs7732671 $\mathrm{G}>\mathrm{C}$ and $P P A R G C 1 B$ rs $17572019 \mathrm{G}>\mathrm{A}$ polymorphisms in gene position significantly increased the risk of T2DM (OR $=1.39,95 \% \mathrm{CI}=1.01-1.90 ; P=0.041$; and $\mathrm{OR}=$ $1.83,95 \%$ CI $=1.22-2.75 ; P=0.003$, respectively). However, CCCCACA haplotype with the same order of polymorphisms in gene position conferred a decreased risk to $\mathrm{T} 2 \mathrm{DM}(P=0.008)$.

\section{Association between PPARGC1A rs3736265 G>A polymorphism and biochemistry characteristics}

As pharmacotherapy for T2DM might affect biochemistry characteristics, in this stage, only nondiabetic controls were enrolled. We evaluated the association of PPARGC1A rs3736265 G>A polymorphism with biochemistry characteristics using Student's t-test. As shown in Table 6, there was a significant correlation of PPARGC1A rs3736265 $\mathrm{G}>\mathrm{A}$ polymorphism with FPG. When the FPG level of PPARGC1A rs3736265 GG genotype was used as the reference group, FPG level of the GA/AA and GA genotypes significantly decreased [GA+AA vs. GG: $P=0.009$ and GA vs. GG: $P=0.002$
(Table 6)]. We also found that PPARGC1A rs3736265 $\mathrm{G}>$ A polymorphism had an association with Triglyceride. When the triglyceride level of PPARGC1A rs3736265 GG genotype was used as the reference group, triglyceride level of the AA genotype significantly increased (AA vs. GG: $P=0.014$, Table 6). When the triglyceride level of PPARGC1A rs3736265 GG/GA genotypes was used as the reference group, triglyceride level of the AA genotype also significantly increased (AA vs. GG/GA: $P=0.017$, Table 6).

\section{DISCUSSION}

T2DM is the most prevalent metabolic diseases worldwide, and it is multi-factorial disorder that results from the interaction of individual's genetic background with environmental factor. Recently, exploration of susceptibility variants has become an important approach to study the etiology of T2DM. Recent studies demonstrated that susceptibility of T2DM could be influenced by variants in some energy balance and lipid /glucose metabolism genes $[18,19]$. We chose $P P A R G$, PPARGC1A, PPARGC1B genes for their impact on glucose metabolism and the biological plausibility of a role in the development of IR $[20,21]$. Using a casecontrol study approach, we investigated relationships of 
Table 6: Associations of the PPARGC1A rs3736265 G>A genetic variants with biochemistry characteristics among control participants

\begin{tabular}{|c|c|c|c|c|c|c|c|c|c|c|c|c|}
\hline \multirow[t]{2}{*}{ Genotype } & \multicolumn{2}{|c|}{$\begin{array}{c}\text { Controls } \\
(n=782)\end{array}$} & \multirow{2}{*}{$\begin{array}{c}\text { FPG } \\
(\mathrm{mmol} / \mathrm{L})\end{array}$} & \multirow[t]{2}{*}{$P$} & \multirow{2}{*}{$\begin{array}{c}\text { Total } \\
\text { cholesterol } \\
(\mathrm{mmol} / \mathrm{L})\end{array}$} & \multirow[t]{2}{*}{$P$} & \multirow{2}{*}{$\begin{array}{c}\text { Triglyceride } \\
\text { (mmol/L) }\end{array}$} & \multirow[t]{2}{*}{$\boldsymbol{P}$} & \multirow{2}{*}{$\begin{array}{c}\text { HDL-C } \\
(\mathrm{mmol} / \mathrm{L})\end{array}$} & \multirow[t]{2}{*}{$P$} & \multirow{2}{*}{$\begin{array}{c}\text { LDL-C } \\
(\mathrm{mmol} / \mathrm{L})\end{array}$} & \multirow[t]{2}{*}{$P$} \\
\hline & $\mathbf{n}$ & $\%$ & & & & & & & & & & \\
\hline GG & 557 & 71.41 & $5.16 \pm 0.47$ & 1.0 & $4.87 \pm 1.02$ & 1.0 & $1.52 \pm 0.93$ & 1.0 & $1.29 \pm 0.35$ & 1.0 & $3.14 \pm 0.82$ & 1.0 \\
\hline GA & 196 & 25.13 & $5.04 \pm 0.50$ & 0.002 & $4.88 \pm 1.00$ & 0.878 & $1.57 \pm 0.97$ & 0.507 & $1.33 \pm 0.39$ & 0.238 & $3.11 \pm 0.79$ & 0.704 \\
\hline AA & 27 & 3.46 & $5.21 \pm 0.66$ & 0.601 & $5.10 \pm 1.22$ & 0.249 & $1.97 \pm 0.95$ & 0.014 & $1.27 \pm 0.46$ & 0.695 & $3.34 \pm 0.92$ & 0.220 \\
\hline $\mathrm{GA}+\mathrm{AA}$ & 223 & 28.59 & $5.06 \pm 0.53$ & 0.009 & $4.91 \pm 1.03$ & 0.624 & $1.62 \pm 0.97$ & 0.179 & $1.32 \pm 0.40$ & 0.337 & $3.14 \pm 0.81$ & 0.980 \\
\hline $\mathrm{GG}+\mathrm{GA}$ & 753 & 96.54 & $5.13 \pm 0.48$ & 1.0 & $4.87 \pm 1.01$ & 1.0 & $1.53 \pm 0.94$ & 1.0 & $1.30 \pm 0.36$ & 1.0 & $3.13 \pm 0.81$ & 1.0 \\
\hline AA & 27 & 3.46 & $5.21 \pm 0.66$ & 0.395 & $5.10 \pm 1.22$ & 0.249 & $1.97 \pm 0.95$ & 0.017 & $1.27 \pm 0.46$ & 0.606 & $3.34 \pm 0.92$ & 0.197 \\
\hline
\end{tabular}

FPG: fasting plasma glucose;

HDL-C, high-density lipoprotein cholesterol;

LDL-C, low-density lipoprotein cholesterol;

Bold values are statistically significant $(P<0.05)$

PPARG, PPARGC1A, PPARGC1B polymorphisms with T2DM. In addition, we studied the association between validated SNP and biochemistry characteristics. In this study, we identified that PPARGC1A rs3736265 G>A polymorphism was associated with the decreased risk of T2DM. We also found PPARGC1A rs3736265 A allele might modulate the level of FPG and serum triglyceride.

PPARG rs1801282 $\mathrm{C}>\mathrm{G}$ polymorphism, a SNP in exon $\mathrm{B}$, encodes a proline (Pro) to alanine (Ala) substitution at amino acid residue [22]. A previous study reported this missense substitution (Pro $\rightarrow$ Ala) might decrease transcriptional activation of PPARG gene in vitro [23]. The other important SNP in PPARG gene, rs3856806 $\mathrm{C}>\mathrm{T}$ polymorphism, is consistently associated with higher BMI, whilst PPARG rs1801282 $\mathrm{C}>\mathrm{G}$ polymorphism is consistently associated with a lower BMI [24]. PPARG rs1801282 C $>\mathrm{G}$ and rs3856806 $\mathrm{C}>\mathrm{T}$ polymorphisms may affect the balance of energy metabolism and cell differentiation, and then presumably alter the susceptibility of T2DM. In this study, as shown in Table 3, PPARG rs $1801282 \mathrm{C}>\mathrm{G}$ polymorphism might not confer the susceptibility to T2DM, which did not agree with results of the previous meta-analysis [25]. However, we found that only three small sample size studies with 1099 T2DM cases and 985 non-diabetic controls were included in that analysis [25]. The evidence might be limited. As for PPARG rs3856806 $\mathrm{C}>\mathrm{T}$ polymorphism, Du et al. [26] and Liu et al. [27] found that this SNP was associated with T2DM in a Chinese population. While Cho et al. [28] reported a negative result in cases with gestational diabetes mellitus in the Korean population, which was similar to our results. Therefore, whether the $\mathrm{C} \rightarrow \mathrm{T}$ transition of $\mathrm{rs} 3856806$ polymorphism in PPARG gene does change biological activity of PPARG protein are needed to be further explored.

PPARGC1A, a transcriptional co-activator of PPARG, regulates transcription in adipogenesis, oxidative metabolism and adaptive thermogenesis relative genes [29]. Recently, some functional studies reported that PPARGC1A also control the restoration of insulinsensitive glucose transporter (Glucose transporter type 4) gene expression in muscle cells [30], gluconeogenesis in liver and as a central target of the hepatic insulin-cAMP axis [31]. Moreover, in muscle, decreased expression of PPARGC1A was observed in diabetes cases and even in non-diabetic individuals with a family history of diabetes [32]. Several epidemiological investigations have explored the effects of PPARGC1A rs8192678G $>$ A (Gly482Ser) and PPARGC1A rs2970847 C>T (Thr394Thr) in exon 8 and PPARGC1A rs3736265 $\mathrm{G}>\mathrm{A}$ (Thr612Met) in exon 9 on the development of T2DM; however, the relationships have not been consistently replicated. For PPARGC1A rs8192678G $>$ A polymorphism, results of some meta-analysis attained consistent findings that this SNP is associated with the increased susceptibility to T2DM in overall populations $[14,15]$. While in a subgroup analysis, this association between PPARGC1A rs8192678G $>$ A polymorphism and T2DM was not observed in east Asians [14], which is analogous to our findings. Previous study suggested that PPARGC1A rs3736265 G>A polymorphism was associated with the decreased risk of T2DM in a Danish population, and the PPARGC1A rs3736265A allele might be a protective factor in T2DM [33]. However, Kim et al. reported that PPARGC1A rs3736265 $\mathrm{G}>\mathrm{A}$ polymorphism was not associated with the risk of T2DM in the Korean population 
[34]. In this study, we also focused on the association between PPARGC1A rs3736265 $\mathrm{G}>\mathrm{A}$ polymorphism and risk of T2DM. We identified that GA/AA and GA genotype of PPARGC1A rs3736265 G>A polymorphism was correlated with a borderline statistically decreased susceptibility of T2DM. In subsroup analyses, we found that PPARGC1A rs3736265 $\mathrm{G}>\mathrm{A}$ polymorphism was associated with decreased risk of T2DM in $<65$ years and female subgroups. As susceptibility SNP for T2DM might affect biochemistry characteristics, we also evaluated the association of PPARGC1A $\mathrm{rs} 3736265 \mathrm{G}>$ A polymorphism with biochemistry characteristics. Results of our studies indicated that PPARGC1A rs3736265 $\mathrm{G}>$ A polymorphism was associated with the decreased level of FPG, while it might increase the level of serum triglyceride. The variants of PPARGC1A rs3736265 $\mathrm{G}>\mathrm{A}$ polymorphism decrease the level of FPG, then they might be a protective factor for the development of T2DM. These associations between PPARGC1A rs $3736265 \mathrm{G}>$ A polymorphism and biochemistry characteristics were consistent with the results of the present case-control study. However, given PPARGC1A rs3736265 G>A polymorphism might have opposite effects on FPG and the level of triglyceride, function of this SNP should be further explored.

A number of studies have highlighted that PPARGC1B plays an important role in regulating energy metabolism including fatty acid oxidation, thermogenesis, gluconeogenesis and mitochondrial biogenesis [13, $31,35,36]$. The human PPARGC1B gene, encoding the PPARGC1B protein, locates on chromosome $5 \mathrm{q} 32$, a relative area that suggests linkage to T2DM [24]. The PPARGC1B rs $7732671 \mathrm{G}>\mathrm{C}$ and $\mathrm{rs} 17572019 \mathrm{G}>\mathrm{A}$ variants were in almost complete linkage disequilibrium in Caucasians $\left(\mathrm{R}^{2}=0.958\right)$ and they were associated with the decreased risk of obesity [16]. A recent GWAS study demonstrated that $P P A R G C 1 B$ rs $7732671 \mathrm{G}>\mathrm{C}$ and rs $17572019 \mathrm{G}>\mathrm{A}$ polymorphisms were not associated with T2DM risk [12]. In this study, the distribution of genotype frequencies of $P P A R G C 1 B$ rs $7732671 \mathrm{G}>\mathrm{C}$ and rs $17572019 \mathrm{G}>\mathrm{A}$ polymorphisms was not significantly different between T2DM cases and the controls. The findings were consistent with the previous GWAS studies mentioned above.

Because PPARG rs $1801282 \quad \mathrm{C}>\mathrm{G}, \quad$ PPARG rs3856806 C $>\mathrm{T}, \quad P P A R G C 1 A \quad$ rs8192678 $\mathrm{C}>\mathrm{T}$, PPARGC1A rs2970847 C>T, PPARGC1A rs3736265 $\mathrm{G}>\mathrm{A}$, PPARGC1B rs7732671 $\mathrm{G}>\mathrm{C}$ and PPARGC1B rs $17572019 \mathrm{G}>\mathrm{A}$ polymorphisms may be not inherited randomly, but as construction of alleles in this study, we harnessed an online program to analyze inherited patterns of the seven SNPs. We found the frequency of CTTCGGG and CTCTGGG haplotypes with the order of PPARG rs $1801282 \mathrm{C}>\mathrm{G}$, PPARG rs3856806 $\mathrm{C}>\mathrm{T}$, PPARGC1A rs8192678 C $>\mathrm{T}$, PPARGC1A rs $2970847 \mathrm{C}>\mathrm{T}$, $P P A R G C 1 A$ rs $3736265 \mathrm{G}>\mathrm{A}, P P A R G C 1 B$ rs $7732671 \mathrm{G}>\mathrm{C}$ and PPARGC1B rs17572019 $\mathrm{G}>\mathrm{A}$ polymorphisms in gene position was significantly increased in T2DM patients. However, CCCCACA haplotype with the same order of polymorphisms may decreased the risk of T2DM. We first reported the association of combined PPARG, PPARGC1A and PPARGC1B haplotypes with T2DM susceptibility.

Using an online Power and Sample Size Calculator (http://biostat.mc.vanderbilt.edu/twiki/bin/view/Main/ PowerSampleSize), the power of the present study was evaluated $(\alpha=0.05)$. For PPARGC1A rs3736265 $\mathrm{G}>\mathrm{A}$ polymorphism, the power was 0.469 in additive model and 0.508 in dominant model among overall T2DM group, 0.919 in additive model and 0.920 in dominant model among female subgroup, and 0.721 in additive model and 0.558 in dominant model among $<65$ years subgroup.

Like all epidemiological case-control studies, some limitations should be taken into account. Firstly, our study was that it was designed as a hospital-based study. All T2DM cases and non-diabetic controls were recruited from two hospitals which located in Eastern China. Although, in our study, the MAF in controls was very similar to the MAF of Chinese in the database (Table 2), the selection bias might have occurred. Secondly, we only selected seven important SNPs in PPARG and PPARGC1 family gene, which might not give an extensive view of the genetic susceptibility in PPARG, PPARGC1A and PPARGC1B. Thirdly, since significant correlation was manly found in subgroups of patients after a stratified analysis, the power of study might be limited. In the future, further studies with large sample sizes and detailed gene-environmental data are need to confirm or refute these results.

In conclusion, to the best of our knowledge, this is the first investigation about the possible correlation between PPARGC1A rs3736265 G>A polymorphism and biochemistry characteristics. Our findings suggest that variants of PPARGC1A rs3736265 $\mathrm{G}>\mathrm{A}$ polymorphism decrease the level of FPG, improving the expectation of study in individual's prevention strategies to T2DM.

\section{MATERIALS AND METHODS}

\section{Subjects}

A total of 1,284 subjects from Eastern Chinese Han population were enrolled for this case-control study. There were 502 T2DM patients and 784 non-diabetic controls. Our study conforms to the items of the Declaration of Helsinki, and was approved by Jiangsu University (Zhenjiang, China) and Fujian Medical University Ethics Committee (Fuzhou, China). T2DM cases were selected from the department of endocrine at the Affiliated People's Hospital of Jiangsu University and the Affiliated Union Hospital of Fujian Medical University, and the controls were recruited from Health Check Centers at these hospitals. All participants provided written informed consent. All subjects were recruited between October 
2014 and May 2016 consecutively. Demographic variables and risk factors of all subjects were collected by two experienced doctors. Anthropometric measurements (e.g. systolic blood pressure, diastolic blood pressure, weight and height) were tested using standard techniques. Body mass index (BMI) was assessed as weight (kilograms) divided by height (meters) squared. Serum triglycerides, total cholesterol, high-density lipoprotein cholesterol (HDL-C), high-density lipoprotein cholesterol (LDL-C), and fasting plasma glucose (FPG) were also measured. In addition, according to the criterion for overweight and obesity, a BMI of 24 was used as the cut-off point in Chinese adults $[37,38]$. The information is listed in Table 1. All experimental protocol was conducted in accordance with the approved guidelines.

The World Health Organization 1999 guidelines of T2DM were used as the criteria for diagnosis [18]. For the eligible controls, the following criteria were used: no history of T2DM, postprandial plasma glucose (PPG) < $7.8 \mathrm{mmol} / \mathrm{L}$ and normoglycemia $[\mathrm{FPG}<6.1 \mathrm{mmol} / \mathrm{l})][19]$.

\section{DNA extraction and genotyping}

Samples of peripheral blood were collected with ethylenediamine tetraacetic acid (EDTA) anticoagulant vacutainer tubes (BD Franklin Lakes NJ, USA). Blood samples were stored at $-20^{\circ} \mathrm{C}$. Genomic DNA was extracted from lymphocytes using the Promega Genomic DNA Purification Kit (Promega, Madison, USA). The genomic DNA obtained was frozen at $-80^{\circ} \mathrm{C}$ for SNP analysis. Genotyping of PPARG rs1801282 $\mathrm{C}>\mathrm{G}$, PPARG rs3856806 C>T, PPARGC1A rs8192678 C $>\mathrm{T}$, $P P A R G C 1 A$ rs $2970847 \mathrm{C}>\mathrm{T}, P P A R G C 1 A$ rs 3736265 $\mathrm{G}>\mathrm{A}, P P A R G C 1 B$ rs7732671 $\mathrm{G}>\mathrm{C}$ and $P P A R G C 1 B$ rs $17572019 \mathrm{G}>\mathrm{A}$ polymorphisms was carried out using the SNPscan ${ }^{\mathrm{TM}}$ genotyping assay (Gnensky Biotechologies Inc., Shanghai, China). The success rate of all genotyping was $>99 \%$ (Table 2). The genotypes of PPARG rs 1801282 $\mathrm{C}>\mathrm{G}, P P A R G$ rs3856806 C $>\mathrm{T}, P P A R G C 1 A$ rs 8192678 $\mathrm{C}>\mathrm{T}, P P A R G C 1 A$ rs $2970847 \mathrm{C}>\mathrm{T}, P P A R G C 1 A$ rs 3736265 $\mathrm{G}>\mathrm{A}, P P A R G C 1 B$ rs7732671 $\mathrm{G}>\mathrm{C}$ and $P P A R G C 1 B$ rs $17572019 \mathrm{G}>\mathrm{A}$ polymorphisms were confirmed by the same DNA genotyping method in fifty-two (4\%) randomly selected samples.

\section{Statistical analysis}

All statistical analyses were performed in SAS 9.4 software (SAS Institute, Cary, NC). The data of continuous variables are expressed as the mean \pm standard deviation (SD). Student's t-test was harnessed to determine the differences for normally distributed continuous variables between T2DM cases and controls. Chi-square test $\left(\chi^{2}\right)$ was conducted to measure the differences for categorical variables (e.g. genotypes, sex, age, smoking status, alcohol use and BMI). We used an internet-based calculator (http://ihg.gsf.de/cgi-bin/hw/hwa1.pl) to measure the Hardy-Weinberg equilibrium (HWE) in controls with the genotype frequencies of $P P A R G$ rs $1801282 \mathrm{C}>\mathrm{G}$, $P P A R G$ rs3856806 C>T, PPARGC1A rs8192678 C $>\mathrm{T}$, PPARGC1A rs2970847 C>T, PPARGC1A rs $3736265 \mathrm{G}>\mathrm{A}$, $P P A R G C 1 B$ rs $7732671 \mathrm{G}>\mathrm{C}$ and PPARGC1B rs 17572019 $\mathrm{G}>\mathrm{A}$ polymorphisms. The associations between $P P A R G$ rs $1801282 \mathrm{C}>\mathrm{G}, P P A R G$ rs $3856806 \mathrm{C}>\mathrm{T}$, PPARGC1A rs8192678 C $>$ T, PPARGC1A rs $2970847 \mathrm{C}>\mathrm{T}, P P A R G C 1 A$ rs3736265 $\mathrm{G}>\mathrm{A}, P P A R G C 1 B$ rs7732671 $\mathrm{G}>\mathrm{C}$ and $P P A R G C 1 B$ rs $17572019 \mathrm{G}>\mathrm{A}$ polymorphisms and risk of T2DM were assessed by crude ORs and adjusted ORs when it was appropriate. SHESIS software (Bio-X Inc., Shanghai, China, http://analysis.bio-X.cn/myAnalysis.php) was used for construction of haplotypes [17]. A $P<0.05$ (two-tailed) was considered as the criterion of statistical significance.

\section{ACKNOWLEDGMENTS}

We appreciate all subjects who participated in this study. We wish to thank Dr. Yan Liu (Genesky Biotechnologies Inc., Shanghai, China) for technical support.

\section{CONFLICTS OF INTEREST}

The authors have no potential financial conflicts of interest.

\section{GRANT SUPPORT}

This study was supported in part by Clinical Medicine Science and Technology Development Fund of Jiangsu University (JLY20140012).

\section{REFERENCES}

1. Xu Y, Wang L, He J, Bi Y, Li M, Wang T, Wang L, Jiang Y, Dai M, Lu J, Xu M, Li Y, Hu N, et al. Prevalence and control of diabetes in Chinese adults. Jama. 2013; 310:948-959.

2. Xi L, Chow CM, Kong X. Role of Tissue and Systemic Hypoxia in Obesity and Type 2 Diabetes. Journal of diabetes research. 2016; 2016:1527852.

3. Sakurai M, Nakamura K, Miura K, Takamura T, Yoshita K, Nagasawa SY, Morikawa Y, Ishizaki M, Kido T, Naruse Y, Nakashima M, Nogawa K, Suwazono Y, et al. Dietary carbohydrate intake, presence of obesity and the incident risk of type 2 diabetes in Japanese men. Journal of diabetes investigation. 2016; 7:343-351.

4. Unger RH, Orci L. Lipoapoptosis: its mechanism and its diseases. Biochimica et biophysica acta. 2002; 1585:202-212. 
5. Manco M, Calvani M, Mingrone G. Effects of dietary fatty acids on insulin sensitivity and secretion. Diabetes, obesity \& metabolism. 2004; 6:402-413.

6. Shimabukuro M, Wang MY, Zhou YT, Newgard CB, Unger RH. Protection against lipoapoptosis of beta cells through leptin-dependent maintenance of Bcl-2 expression. Proceedings of the National Academy of Sciences of the United States of America. 1998; 95:9558-9561.

7. Medina-Gomez G, Gray S, Vidal-Puig A. Adipogenesis and lipotoxicity: role of peroxisome proliferatoractivated receptor gamma (PPARgamma) and PPARgammacoactivator-1 (PGC1). Public health nutrition. 2007; 10:1132-1137.

8. Tonjes A, Stumvoll M. The role of the Pro12Ala polymorphism in peroxisome proliferator-activated receptor gamma in diabetes risk. Current opinion in clinical nutrition and metabolic care. 2007; 10:410-414.

9. Scott LJ, Mohlke KL, Bonnycastle LL, Willer CJ, Li Y, Duren WL, Erdos MR, Stringham HM, Chines PS, Jackson AU, Prokunina-Olsson L, Ding CJ, Swift AJ, et al. A genome-wide association study of type 2 diabetes in Finns detects multiple susceptibility variants. Science. 2007; 316:1341-1345.

10. Berhouma R, Kouidhi S, Ammar M, Abid H, Baroudi T, Ennafaa H, Benammar-Elgaaied A. Genetic susceptibility to type 2 diabetes: a global meta-analysis studying the genetic differences in Tunisian populations. Human biology. 2012; 84:423-435.

11. Al-Rubeaan K, Siddiqui K, Saeb AT, Nazir N, Al-Naqeb D, Al-Qasim S. ACE I/D and MTHFR C677T polymorphisms are significantly associated with type 2 diabetes in Arab ethnicity: a meta-analysis. Gene. 2013; 520:166-177.

12. Villegas R, Williams SM, Gao YT, Long J, Shi J, Cai H, Li H, Chen CC, Tai ES, Consortium ATD, Hu F, Cai Q, Zheng $\mathrm{W}$, et al. Genetic variation in the peroxisome proliferatoractivated receptor (PPAR) and peroxisome proliferatoractivated receptor gamma co-activator 1 (PGC1) gene families and type 2 diabetes. Annals of human genetics. 2014; 78:23-32.

13. Puigserver $\mathrm{P}, \mathrm{Wu} \mathrm{Z}$, Park $\mathrm{CW}$, Graves $\mathrm{R}$, Wright $\mathrm{M}$, Spiegelman BM. A cold-inducible coactivator of nuclear receptors linked to adaptive thermogenesis. Cell. 1998; 92:829-839.

14. Yang Y, Mo X, Chen S, Lu X, Gu D. Association of peroxisome proliferator-activated receptor gamma coactivator 1 alpha (PPARGC1A) gene polymorphisms and type 2 diabetes mellitus: a meta-analysis. Diabetes/ metabolism research and reviews. 2011; 27:177-184.

15. Barroso I, Luan J, Sandhu MS, Franks PW, Crowley V, Schafer AJ, O'Rahilly S, Wareham NJ. Meta-analysis of the Gly482Ser variant in PPARGC1A in type 2 diabetes and related phenotypes. Diabetologia. 2006; 49:501-505.

16. Andersen G, Wegner L, Yanagisawa K, Rose CS, Lin J, Glumer C, Drivsholm T, Borch-Johnsen K, Jorgensen T,
Hansen T, Spiegelman BM, Pedersen O. Evidence of an association between genetic variation of the coactivator PGC-1beta and obesity. Journal of medical genetics. 2005; 42:402-407.

17. Shi YY, He L. SHEsis, a powerful software platform for analyses of linkage disequilibrium, haplotype construction, and genetic association at polymorphism loci. Cell research. 2005; 15:97-98.

18. Yao M, Wu Y, Fang Q, Sun L, Li T, Qiao H. Association of ADIPOQ Variants with Type 2 Diabetes Mellitus Susceptibility in Ethnic Han Chinese from Northeast China. Journal of diabetes investigation. 2016.

19. Fan Y, Li X, Zhang Y, Fan X, Zhang N, Zheng H, Song Y, Shen C, Shen J, Ren F, Yang J. Genetic Variants of TPCN2 Associated with Type 2 Diabetes Risk in the Chinese Population. PloS one. 2016; 11:e0149614.

20. Heim M, Johnson J, Boess F, Bendik I, Weber P, Hunziker W, Fluhmann B. Phytanic acid, a natural peroxisome proliferator-activated receptor (PPAR) agonist, regulates glucose metabolism in rat primary hepatocytes. FASEB journal. 2002; 16:718-720.

21. Ludtke A, Buettner J, Wu W, Muchir A, Schroeter A, ZinnJustin S, Spuler S, Schmidt HH, Worman HJ. Peroxisome proliferator-activated receptor-gamma C190S mutation causes partial lipodystrophy. The Journal of clinical endocrinology and metabolism. 2007; 92:2248-2255.

22. Yen CJ, Beamer BA, Negri C, Silver K, Brown KA, Yarnall DP, Burns DK, Roth J, Shuldiner AR. Molecular scanning of the human peroxisome proliferator activated receptor gamma (hPPAR gamma) gene in diabetic Caucasians: identification of a Pro12Ala PPAR gamma 2 missense mutation. Biochemical and biophysical research communications. 1997; 241:270-274.

23. Masugi J, Tamori Y, Mori H, Koike T, Kasuga M. Inhibitory effect of a proline-to-alanine substitution at codon 12 of peroxisome proliferator-activated receptorgamma 2 on thiazolidinedione-induced adipogenesis. Biochemical and biophysical research communications. 2000; 268:178-182.

24. Doney A, Fischer B, Frew D, Cumming A, Flavell DM, World M, Montgomery HE, Boyle D, Morris A, Palmer CN. Haplotype analysis of the PPARgamma Pro12Ala and C1431T variants reveals opposing associations with body weight. BMC genetics. 2002; 3:21.

25. Wang C, Li X, Huang Z, Qian J. Quantitative assessment of the influence of PPARG P12A polymorphism on gestational diabetes mellitus risk. Molecular biology reports. 2013; 40:811-817.

26. Du J, Shi H, Lu Y, Du W, Cao Y, Li Q, Ma J, Ye X, Cheng J, Yu X, Gao Y, Zhou L. Tagging single nucleotide polymorphisms in the PPAR-gamma and RXR-alpha gene and type 2 diabetes risk: a case-control study of a Chinese Han population. Journal of biomedical research. 2011; 25:33-41. 
27. Lu Y, Ye X, Cao Y, Li Q, Yu X, Cheng J, Gao Y, Ma J, $\mathrm{Du} \mathrm{W}$, Zhou L. Genetic variants in peroxisome proliferatoractivated receptor-gamma and retinoid $\mathrm{X}$ receptoralpha gene and type 2 diabetes risk: a case-control study of a Chinese Han population. Diabetes technology \& therapeutics. 2011; 13:157-164.

28. Cho YM, Kim TH, Lim S, Choi SH, Shin HD, Lee HK, Park KS, Jang HC. Type 2 diabetes-associated genetic variants discovered in the recent genome-wide association studies are related to gestational diabetes mellitus in the Korean population. Diabetologia. 2009; 52:253-261.

29. Puigserver P, Spiegelman BM. Peroxisome proliferatoractivated receptor-gamma coactivator 1 alpha (PGC-1 alpha): transcriptional coactivator and metabolic regulator. Endocrine reviews. 2003; 24:78-90.

30. Michael LF, Wu Z, Cheatham RB, Puigserver P, Adelmant G, Lehman JJ, Kelly DP, Spiegelman BM. Restoration of insulin-sensitive glucose transporter (GLUT4) gene expression in muscle cells by the transcriptional coactivator PGC-1. Proceedings of the National Academy of Sciences of the United States of America. 2001; 98:3820-3825.

31. Yoon JC, Puigserver P, Chen G, Donovan J, Wu Z, Rhee J, Adelmant G, Stafford J, Kahn CR, Granner DK, Newgard $\mathrm{CB}$, Spiegelman BM. Control of hepatic gluconeogenesis through the transcriptional coactivator PGC-1. Nature. 2001; 413:131-138.

32. Patti ME, Butte AJ, Crunkhorn S, Cusi K, Berria R, Kashyap S, Miyazaki Y, Kohane I, Costello M, Saccone R, Landaker EJ, Goldfine AB, Mun E, et al. Coordinated reduction of genes of oxidative metabolism in humans with insulin resistance and diabetes: Potential role of PGC1 and
NRF1. Proceedings of the National Academy of Sciences of the United States of America. 2003; 100:8466-8471.

33. Ek J, Andersen G, Urhammer SA, Gaede PH, Drivsholm T, Borch-Johnsen K, Hansen T, Pedersen O. Mutation analysis of peroxisome proliferator-activated receptor-gamma coactivator-1 (PGC-1) and relationships of identified amino acid polymorphisms to Type II diabetes mellitus. Diabetologia. 2001; 44:2220-2226.

34. Kim JH, Shin HD, Park BL, Cho YM, Kim SY, Lee HK, Park KS. Peroxisome proliferator-activated receptor gamma coactivator 1 alpha promoter polymorphisms are associated with early-onset type 2 diabetes mellitus in the Korean population. Diabetologia. 2005; 48:1323-1330.

35. Lehman JJ, Barger PM, Kovacs A, Saffitz JE, Medeiros DM, Kelly DP. Peroxisome proliferator-activated receptor gamma coactivator-1 promotes cardiac mitochondrial biogenesis. The Journal of clinical investigation. 2000; 106:847-856.

36. Vega RB, Huss JM, Kelly DP. The coactivator PGC-1 cooperates with peroxisome proliferator-activated receptor alpha in transcriptional control of nuclear genes encoding mitochondrial fatty acid oxidation enzymes. Molecular and cellular biology. 2000; 20:1868-1876.

37. Zhai Y, Zhao WH, Chen CM. Verification on the cut-offs of waist circumference for defining central obesity in Chinese elderly and tall adults. [Article in Chinese] Zhonghua liuxingbingxue zazhi. 2010; 31:621-625.

38. Zhang X, Zhang S, Li Y, Detrano RC, Chen K, Li X, Zhao L, Benjamin EJ, Wu Y. Association of obesity and atrial fibrillation among middle-aged and elderly Chinese. International journal of obesity. 2009; 33:1318-1325. 